\title{
Astérisque
}

\section{G. GallavotTi}

\section{Classical KMS condition and Tomita-Takesaki theory}

Astérisque, tome 40 (1976), p. 89-94

<http://www.numdam.org/item?id=AST_1976_40_89_0>

(C) Société mathématique de France, 1976, tous droits réservés.

L'accès aux archives de la collection « Astérisque » (http://smf4.emath.fr/ Publications/Asterisque/) implique l'accord avec les conditions générales d'utilisation (http://www.numdam.org/conditions). Toute utilisation commerciale ou impression systématique est constitutive d'une infraction pénale. Toute copie ou impression de ce fichier doit contenir la présente mention de copyright.

\section{NumDam}

Article numérisé dans le cadre du programme Numérisation de documents anciens mathématiques http://www.numdam.org/ 
Société Mathématique de France

Astérisque 40 (1976) p.89-94

\section{CLASSICAL KMS CONDITION AND TOMITA-TAKESAKI THEORY}

\section{G. GALLAVOTTI}

(Istituto Matematico, Universita di Roma, Italia)

The following structure arises in the theory of quantum statistical mechanics : a pair $(a, p)$ is given consisting in an involutive algebra $a$ which is the inductive union of an increasing family of $C^{*}$-algebras with unity and $\rho$ is a state on $G$ i.e. a positive normalized functional on it.

Among the states $\rho$ there are some which play a special role and which in the physical applications are called "equilibrium states".

To introduce the mathematical definition of the equilibrium states remark that the structure of the algebra $Q$ allows to construct a cyclic *-representation $\pi_{\rho}: Q \rightarrow B(h)$ via the GNS construction. We shall denote $\xi$ the cyclic vector and $\pi_{\rho}(A)$ the image of $A \in Q$ in $B(h)$.

Then the following class of states was suggested naturally for mathematical study by mathematical physics.

Definition. A state $\rho$ is an equilibrium state for some hamiltonian or, briefly, is KMS if

(i) A quadratic form is defined on $\pi_{\rho}(A) \xi \times \pi_{\rho}(A) \xi$ by

$$
\begin{gathered}
\psi\left(\pi_{\rho}(A) \xi, \pi_{\rho}(B) \xi\right)=\rho\left(B A^{*}\right) \\
\text { (i.e. } \left.\rho\left(A^{*} A\right)=0 \Longleftrightarrow \rho\left(A A^{*}\right)=0\right)
\end{gathered}
$$


and defines a positive symmetric operator D such that

$$
\begin{aligned}
& \psi\left(\pi_{\rho}(\mathrm{A}) \xi, \pi_{\rho}(\mathrm{B}) \xi\right)=\left(\mathrm{D}_{\rho}\left(\mathrm{B}^{*}\right) \xi, \mathrm{D}_{\rho}\left(\mathrm{A}^{*}\right) \xi\right) \\
& \text { (ii) the closure of } \mathrm{D} \text { is essentially s.a. on } \pi_{\rho}(\mathrm{Q}) \xi \\
& \text { (i.e. the form } \psi \text { is closable). }
\end{aligned}
$$

I shall not enter into the reasons why the above two mathenatical conditions are related to the characterisation of the equilibrium states in quantum statistical mechanics.

I shall rather describe some properties of the KMS states on an algebra $G$ of the above type. A most remarkable theorem is the following.

\section{Theorem.}

If $\rho$ is a KMS state and $R$ is the Von Neumann algebra generated by $\pi_{0}(O)$ then $D>0$ and $D^{i t} R D^{-i t}=R \quad D^{i t} \xi=\xi$

hence the $\operatorname{map} R \rightarrow \alpha_{t}(R)=D^{i t} R D^{-i t}, R \in R$ is an automorphism of $R$.

This theorem allows to associate to each KMS state a group of automorphisms of $R$ which is called the "time-evolution group" associated to the stationary state $\rho$ and its generator $\mathrm{H}=\log \mathrm{D}$ has, in the physical interpretation, something to do with the hamiltonian of the system.

The interest of the above definition lies, among other things, in the fact that, because of the above theorem, to every KMS (equilibrium) state is automatically associated what is, by the mathematical physicists, considered a partially satisfactory time evolution for the observables which makes the state stationary. 
From a mathematical point of view the above, theorem is a simple corollary of the Tomita-Takesaki theorem.

Theorem.

Let $R$ be a Von Neumann algebra on a Hilbert space $h$ assume that there exists a vector $\xi \in h$ which is cyclic and separating for $R$. Then the quadratic form $\psi$ defined on $\mathbb{R}_{\xi} \times R_{\xi}$ by

$$
\psi(A \xi, B \xi)=\left(B^{*} \xi, A^{*} \xi\right)
$$

is closable and the associated operator $D$ is such that $0>0$ and

$$
D^{i t} R D^{-i t}=R
$$

Viceversa, the Tomita-Takesaki theorem is a consequence of theorem 1 .

The physical situation can be perhaps better understood by considering classical systems on which the mathematicians have often more intuition.

In classical statistical mechanics one is naturally given a *-algebra $Q$ of bounded smooth functions on a topological space $K$; the state $\rho$ is now a Lebesgue measure on $K$ such that $G$ is dense in $L_{2}(\rho)$.

A naive interpretation of the KMS condition can be easily given in this case. It is easily seen, however, that in this case the conditions $i)$, ii) are automatically satisfied and the operator $D$ is just the identify.

In other words this naive formulation of the KMS condition in classical mechanics is totally trivial and deprived of any interest what so ever. 
As I said the above definition of KMS state is natural from a physical point of view in the study of quantum statistical mechanics and, therefore, it is clear that to find the proper formulation for the classical KMS condition one needs to go back to the physical interpretation of the theory.

A simple way of finding out the "classical KMS condition" is to remember that in quantum mechanics there appears an arbitrary constant $\hbar$ and almost all interesting properties of the quantum systems have a non trivial sense in the limit as $\hbar \rightarrow 0$.

If one goes back to the physical interpretation of the quantum statistical mechanics one realizes that the constant $\hbar$ is hidden in several places : in fact, for instance, we had called the parameter $t$ appearing in the theorem 1 the "time" : it is related to the "physical" time $T$ by the relation $t=\frac{T}{h}$ so the value of the observable $\pi_{\rho}(A)$ at time $T$ is

$$
\mathrm{D}^{i \frac{T}{\hbar}} \pi_{\rho} \text { (A) } \mathrm{D}^{-i \frac{T}{\hbar}}
$$

and we see that to obtain the classical time behaviour of the observable $A$ the limit $\hbar \rightarrow O$ has to be taken at fixed $T$ which is completely unclear what it could mean.

However the physicists have developed a rather powerful technique to take these kind of limits "by inspection". I do not enter into the above heuristic considerations. The first is that putting $\hbar$ all over the appropriate places and letting $\hbar \rightarrow 0$ it turns out heuristically that the algebra $Q$ of the observables has some further structure : namely on $Q$ is defined a "Poisson bracket" 
operation which is defined, abstractly, as a map of $Q \times Q$ into smooth functions on $K$ such that
a) $\{f, g\}=-\{g, f\}=\{\overline{\bar{f}, \bar{g}}\}$
b) $\{f, g h\}=\{f, g\} h+\{f, h\} g$

and the KMS condition which is the natural limit as $\hbar \rightarrow 0$ of the above quantum KMS condition is the following :

i) the quadratic form on $a \times a$

$$
\psi(f, g)=\rho(\{\bar{f}, g\})
$$

defines an emisymmetric operator $\mathscr{L}$ ou $\mathrm{L}_{2}(\rho)$ with

domain $C$ such that

$$
\psi(f, g)=\int \overline{(\mathscr{L} f)} g d \rho
$$

ii) the closure of the operator $\mathscr{L}$ is essentially antiselfadjoint on $a$.

The interest of the above definition, besides its formal analogy with the quantum KMS condition, lies in the following theorem

Theorem 3.

If $\rho$ is a KMS state $\exists$ a flow $S_{t}$ on $(K, p)$ such that $\forall f \in L_{\infty}(0)$

$$
\left(e^{\bar{L} t} f\right)(x)=\sigma\left(s_{t} x\right)
$$

This theorem is a consequence and probably, equivalent in some sense to the following more abstract result.

\section{Theorem 4.}

Let $\mathcal{L}$ be an operator on $L_{2}(0)$ which is essentially antiselfadjoint on an algebra $G C L_{\infty}|\rho|$ and which is a derivation on $G$. There exists a flow $s_{t}$ on $(K, p)$ 
such that

$$
\left(e^{\mathscr{L} t} f\right)(x)=6\left(s_{t} x\right) .
$$

This last theorem seems of interest in itself and it seems to have not yet been explicitely remarked in the literature. Its proof is strikingly similar to the proof of the theorem 2 of Tomita-Takesaki. Heuristically it can be obtained by introducing the arbitrary constant $\hbar$ and by wisely letting it tend to zero in all the crucial steps of the proof of the theorem of Tomita-Takesaki.

It seems possible that many interesting concepts and theorems of the theory of the Von Neumann algebras may be carried to function algebras with Poisson bracket structure. The role of the theorem of Tomita-Takesaki should now be played by the above two theorems and the correspondence principle should be a powerful heuristic tool. 